\title{
CONTEÚDO INFORMACIONAL DAS PREVISÕES DE LUCRO DOS ANALISTAS DE MER- CADO E DOS MODELOS DE PREVISÃO RANDOM WALK NO BRASIL ${ }^{1}$
}

\section{INFORMATIONAL CONTENT OF MARKET ANALYSTS' PROFIT FORECASTS AND RANDOM WALK FORECASTING MODELS IN BRAZIL}

\author{
Rafael Confetti Gatsios \\ Doutor em Ciências Contábeis (USP-RP) \\ Universidade de São Paulo (FEARP-USP) \\ rafaelgatsios@hotmail.com \\ Fabiano Guasti Lima \\ Doutorado em Administração (USP) \\ Universidade de São Paulo (USP) \\ fabianoguastilima@gmail.com \\ Rafael Moreira Antônio \\ Doutorado em Controladoria e Contabilidade (USP) \\ rafael.antonio@usp.br \\ Bruno Figlioli \\ Doutor em Ciências Contábeis (FEAUSP-RP) \\ figlioli@usp.br
}

\section{RESUMO}

Objetivo: Este trabalho avalia as previsões de lucro dos analistas e dos modelos random walk, simples e com crescimento, a curto e longo prazos, para as empresas brasileiras de capital aberto entre o período de 2010 e 2015.

Fundamento: A pesquisa apresenta um estudo sobre o conteúdo informacional das previsões de lucros dos analistas de mercado e dos modelos random walk na previsão de resultados futuros das empresas brasileiras de capital aberto a curto e longo prazos.

Método: Os dados foram obtidos via plataforma da ${\text { Thomson } \text { Reuters }^{\circledR} \text {, nas bases de dados I/B/E/S }}^{\circledR}$ e Thomson Financial ${ }^{\circledR}$. Para análise dos resultados foi utilizada a metodologia de regressão linear simples (OLS) robusta a heterocedasticidade.

Resultados: A análise dos modelos sobre o conteúdo informacional aponta maior relevância das previsões random walk com relação às previsões dos analistas. Adicionalmente nota-se que o conteúdo informacional das previsões dos analistas vai perdendo intensidade com o aumento da defasagem da previsão. Destaca-se ainda, um maior conteúdo informacional das previsões dos modelos random walk, mesmo para previsões de curto prazo.

\footnotetext{
${ }^{1}$ Artigo recebido em: 19/09/2019. Revisado por pares em: 28/01/2020. Reformulado em: 16/03/2020. Recomendado para publicação: 11/03/2020 por Anna Paola Fernandes Freire (Editora Adjunta). Publicado em: 25/05/2020. Organização responsável pelo periódico: UFPB 
Contribuições: A principal contribuição deste estudo é a de apontar uma ineficiência nas previsões de lucros dos analistas de mercado, especialmente quando essas previsões são comparadas àquelas obtidas por meio de modelos de séries temporais. Além disso, os resultados apontam a utilidade nos modelos random walk que podem ser usados tanto por analistas como por investidores, sejam eles institucionais ou não.

Palavras-chave: Analistas de mercado. Random walk. Previsão de Lucro. Superioridade dos Analistas. Conteúdo Informacional das Previsões de Lucro.

\section{ABSTRACT}

Objective: This paper evaluates the profit forecasts of the analysts and the simple and growing short walk and long term random walk models for publicly traded Brazilian companies from 2010 to 2015.

Background: The research presents a study on the informational content of market analysts' profit forecasts and random walk models in predicting future results of Brazilian public companies in the short and long term.

Method: Data were obtained via the Thomson Reuters ${ }^{\circledR}$ platform from the $\mathrm{I} / \mathrm{B} / \mathrm{E} / \mathrm{S}^{\circledR}$ and Thomson Financial ${ }^{\circledR}$ databases. For the analysis of the results, the simple linear regression methodology (OLS) robust to heteroscedasticity was used.

Results: The analysis of the models on informational content points to the greater relevance of random walk forecasts in relation to analysts 'forecasts. In addition, it is noted that the informational content of analysts' forecasts decreases in intensity as the forecast lag increases. Also noteworthy is the greater information content of the predictions of random walk models, even for short-term forecasts.

Contributions: The main contribution of this study is to point to an inefficiency in market analysts' earnings forecasts, especially when those forecasts are compared to those obtained through time series models. Moreover, the results point to the utility in random walk models that can be used by both analysts and investors, whether institutional or not.

Keywords: Market Analysts. Random walk. Profit Forecast. Superiority of Analysts. Earnings Forecast Informational Content.

\section{INTRODUÇÃO}

Os analistas são importantes para o mercado de capitais, pois mesmo antes da divulgação do resultado, fornecem um consenso sobre esse valor a ser reportado pela empresa, além de emitirem recomendações de ações e relatórios de empresas específicas e de setores (Antônio, Lima \& Pimenta Junior (2015)). As mesas de operações utilizam as previsões de lucros dos analistas na avaliação do resultado real, reforçando assim a afirmação de Boff, Procianoy e Hoppen (2006), que colocam como principal trabalho dos analistas a interpretação das informações divulgadas pelas empresas. Para Antônio, Rathke, Moraes e Ambrozini (2018) os analistas atuam como intermediários das informações no mercado de capitais e reduzem a assimetria informacional, contribuindo para e eficiência do mercado de capitais.

Há dois tipos de analistas, o buy side, que avalia as opções de investimentos para um determinado banco ou corretora formar sua carteira própria de investimentos, enquanto o sell side é o que faz recomendações de investimentos para um público mais abrangente, que podem ser investidores institucionais e pequenos investidores. No entanto, são formas de atuações distintas, uma vez que o analista sell side pode contribuir sobretudo para aumentar a captação de clientes para determinada corretora a qual ele está vinculado. 
A partir das previsões de lucros, o mercado passa a avaliar o lucro da empresa em comparação a esse valor previsto, e as mesas de operações tomam decisões com base na comparação entre o consenso de mercado e o valor reportado pela companhia, sendo essa diferença fator fundamental para antever o comportamento do ativo no mercado de capitais (Martinez (2004)). Ainda de acordo com Martinez (2004), as empresas que apresentam resultados superiores às previsões dos analistas possuem retorno superior às demais empresas no mercado pelo período de 30 dias. Conforme apresentado por Lima (2011), as previsões mais precisas reduzem a incerteza na tomada de decisão.

De maneira complementar aos estudos apresentados sobre a qualidade das previsões dos analistas, a literatura internacional compara a previsão de resultado pelos modelos random walk com as previsões dos analistas de mercado. Nas décadas de 1970 e 1980 esses estudos foram recorrentes na literatura, ocorrendo uma migração posterior para a avaliação dos fatores que influenciavam na maior ou menor superioridade dos analistas de mercado na previsão de resultado das empresas, buscando os determinantes dessa superioridade (Brown, Hagerman, Griffin \& Zmijewski, 1982, Brown, Richardson \& Schwager, 1982; Fried \& Givoly, 1982; Hopwood \& Mckeown, 1982; O'brien, 1988). Brown et al. (1982a), em estudo seminal sobre o tema, concluíram que as previsões dos analistas de mercado foram superiores aos modelos de séries temporais e indicaram que os analistas possuem vantagens com relação aos modelos de séries temporais, destacando a vantagem informacional e a vantagem relacionada ao tempo.

Mais recentemente, os estudos passaram a reavaliar a superioridade dos analistas de mercado e a discutir quando e sobre quais circunstâncias as estimativas dos analistas foram superiores às previsões dos modelos de séries temporais (Ball \& Ghysels, 2017; Bradshaw et al., 2012; Lacina, Lee \& Xu, 2011; Lorek \& Pagach, 2014). A literatura atual ainda investiga o conteúdo informacional das previsões dos analistas de mercado e dos modelos random walk. O conteúdo informacional está relacionado à capacidade e assertividade nas previsões de lucros, e Bueno (2012) destacou que o modelo random walk é estabelecido como um passeio aleatório de tendência estocástica pura, sendo, portanto, um processo não estacionário. A partir de um modelo random walk entende-se que a melhor estimativa para o resultado futuro é o próprio valor presente. Cheng, Fan e So (2003) e Lacina, Lee e Xu (2011) apontaram que as previsões de resultados dos modelos de séries temporais apresentam maior informação com relação ao lucro atual das empresas, resultado que está relacionado à persistência dos lucros.

No contexto brasileiro, as pesquisas sobre previsão de resultados são majoritariamente sobre as projeções realizadas pelos analistas de mercado, sendo que poucas abordam a utilização de modelos de séries temporais (Silva, 1998; Martinez, 2004, 2007; Dalmácio, Lopes \& Sarlo Neto, 2013; Lima, 2013; Gatsios \& Lima, 2014; Martinez \& Dumer, 2014; Lima \& Almeida, 2015; Gatsios, Lima \& Assaf Neto, 2016; Antônio, Lima \& Santos., 2019).

O trabalho de Lima (2013) apresenta os principais estudos na área de previsão de analistas para o mercado Brasileiro. A pesquisa aponta para uma pequena quantidade de estudos na área, sem consistência na evolução ao longo dos anos e concentradas nos determinantes da acurácia, dispersão e viés das estimativas dos analistas. Ainda, de maneira comparativa, os resultados indicam baixa qualidade das estimativas para o lucro das empresas brasileiras frente aos resultados apresentados para os mercados internacionais, sendo essas previsões menos acuradas, com alta dispersão e com predomínio de viés positivo (Dalmácio, Lopes \& Sarlo Neto, 2013; Gatsios \& Lima, 2014; Martinez, 2004, 2007). Por fim, Gatsios e Lima (2019) apontaram que as previsões dos analistas de mercado são mais acuradas apenas para defasagens curtas, de três meses. Segundo os autores, para os períodos de previsões mais longos verifica-se a maior capacidade preditiva dos lucros para os modelos random walk. 
Assim, apresentada a literatura sobre o conteúdo informacional das previsões de lucro e dada a baixa qualidade preditiva dos analistas de mercado na previsão de resultados futuros e ainda as características do contexto mercado de capitais brasileiro, que apresenta reduzida quantidade de empresas listadas, baixo número de analistas atuando na avaliação das empresas e elevada concentração acionária, apresenta-se a discussão sobre o conteúdo informacional das previsões dos analistas de mercado e a comparação desse conteúdo informacional com previsões realizadas por modelos random walk para o caso brasileiro. Dadas as características apresentadas anteriormente, os resultados podem ser divergentes dos achados na literatura internacional sobre o tema.

Com isso, o trabalho busca responder ao seguinte questionamento: qual o conteúdo informacional das previsões dos analistas de mercado e dos modelos random walk na previsão de lucro das empresas brasileiras não financeiras de capital aberto?

Para responder ao problema de pesquisa é apresentado um modelo de regressão linear simples proposto por Cheng, Fan e So (2003) e Lacina, Lee e Xu (2011) para avaliar o conteúdo informacional das previsões dos analistas e dos modelos random walk no período de 2010 a 2015, com defasagens de curto e longo prazos. A análise dos modelos sobre o conteúdo informacional aponta maior relevância das previsões random walk quando comparadas às previsões dos analistas. Além disso, os resultados indicaram que o conteúdo informacional obtido a partir das previsões dos analistas perde intensidade com o aumento da defasagem dessas previsões. Destaca-se ainda, um maior conteúdo informacional das previsões dos modelos mesmo para previsões de curto prazo. Estes resultados sugerem baixa eficiência dos analistas de mercado na redução da assimetria informacional no mercado de capitais brasileiro.

\section{REFERENCIAL TEÓRICO}

\subsection{Analistas de Mercado}

Ramnath, Rock e Shane (2008) avaliaram a literatura de analistas de mercado e apresentam uma classificação sobre as linhas de estudo deste tema de pesquisa. Nessa revisão da literatura realizada por Ramnath, Rock e Shane (2008), é desenvolvida uma taxonomia de pesquisa para examinar o papel dos analistas, e os autores partiram das perspectivas fornecidas por Schipper (1991) e Brown (1993). A partir disso, obtiveram 250 artigos publicados desde 1992 nas 11 maiores revistas. Os periódicos analisados foram: The Accounting Review, Contemporary Accounting Research, International Journal of Forecasting, Journal of Accounting and Economics, Journal of Accounting Research, Journal of Business, Journal of Finance, Journal of Financial Economics, Journal of Financial and Quantitative Analysis, Review of Accounting Studies, and Review of Financial Studies.

A classificação apresentada pelos autores divide os estudos sobre os analistas em sete categorias, de acordo com o objetivo do estudo. As áreas destacadas são: processo de decisão dos analistas; características dos analistas; experiência e distribuição das previsões desses agentes; informação contida nos relatórios divulgados pelos analistas; relação entre analistas e eficiência de mercado; pesquisas sobre viés de previsão; impacto de alterações regulatórias nas previsões dos analistas; e desenhos de pesquisa e fonte de dados.

Kothari (2001) apresenta uma discussão sobre os analistas, indicando que a linha de pesquisa é utilizada para avaliação de questões práticas e teóricas no mercado de capitais. $\mathrm{O}$ autor aponta que as pesquisas são essencialmente utilizadas para entender as previsões dos analistas e seus determinantes, baseadas fundamentalmente nas previsões dos analistas sell-side; uma vez que, para esse grupo de agentes, há informações disponíveis no mercado, ao contrário da categoria buyside, que realiza projeções para departamentos internos.

Jegadeesh, Krische e Lee (2004) apontam que os pesquisadores e os participantes do mercado se interessam em compreender como as atividades dos analistas afetam a eficiência do mercado de capitais. Neste ponto é importante avaliar o poder dos analistas de mercado na redução da as- 
simetria informacional, uma vez que esses atuam como intermediários das informações contábeis e financeiras das empresas para os investidores, como apresentado por Antônio, Rathke, Moraes e Ambrozini (2018). Nesse sentido, quanto maior o conteúdo informacional das previsões dos analistas para o lucro das empresas, maior a transferência de informações para os agentes, e consequentemente, mais eficientes os mercados de acordo com Fama (1970). Nessa linha, Hall e Tacon (2010) destacaram que os analistas reduzem a assimetria informacional promovem eficiência de mercado.

\subsection{Superioridade dos Analistas}

A literatura relacionada à superioridade na previsão dos analistas apresenta estudos que compararam as previsões dos analistas de mercado com modelos simples de previsão, enquanto alguns autores empregam outros modelos de séries temporais para avaliar a superioridade dos analistas. Ao longo das décadas de 1970 e 1980 esses estudos foram recorrentes na literatura, após esse período começaram a ser avaliados os fatores que influenciaram na maior (ou menor) superioridade dos analistas em suas previsões de resultados das empresas, buscando os determinantes dessa superioridade (Brown, Hagerman, Griffin \& Zmijewski, 1982, Brown, Richardson \& Schwager, 1982; Fried \& Givoly, 1982; Hopwood \& Mckeown, 1982; O'brien, 1988).

Brown et al. (1982a) em estudo seminal sobre o tema, concluíram que as previsões dos analistas são superiores àquelas obtidas por meio dos modelos de séries temporais. Esse estudo foi complementado por pesquisa semelhante, que indicou que os analistas possuíram vantagens informacionais e temporais com relação aos modelos de séries temporais (Brown et al., 1982a, 1982b). Com base nos resultados desses dois principais estudos da sobre o tema, a literatura passou a atribuir a superioridade dos analistas a dois aspectos: i) vantagem informacional e ii) vantagem temporal. Para Brown et al. (1982a, 1982b), a vantagem informacional se deve ao fato de os analistas possuírem maior conteúdo informacional para a realização das estimativas do que os modelos de séries temporais. Essas informações são relacionadas à empresa, ao setor, ao mercado de capitais e ainda informações econômicas, o que denota um maior conjunto informacional a ser utilizado e incorporado nas previsões.

A segunda vantagem está relacionada ao tempo de realização das previsões, ou seja, os analistas podem realizar estimativas logo após a divulgação dos resultados da empresa. Enquanto isso, os modelos de séries temporais utilizam apenas os dados passados. Essa vantagem temporal permite aos analistas agregar mais informações ao longo do processo de previsão de resultado. Neste sentido, esses resultados apontam para um conteúdo informacional relevante das previsões dos analistas, ou seja, os analistas de mercado são relevantes para a disseminação de informações, contribuindo assim para o aumento da eficiência dos mercados.

Após os resultados obtidos pelos trabalhos de Brown et al. (1982a, 1982b), a linha de pesquisa que compara a acurácia dos analistas com as previsões dos modelos de séries temporais foi deixada de lado, com a impressão de que já se havia chegado a uma conclusão definitiva sobre o tema, com poucos estudos apresentados na década de 1990 (Kross, Ro \& Schroeder, 1990; Brown, 1993; Branson, Lorek \& Pagach, 1995; Silva, 1998). No contexto brasileiro, Silva (1998) avaliou as empresas brasileiras de capital aberto no período de 1994 a 1996, e os resultados indicaram que os analistas são mais acurados do que os modelos de séries temporais em estimativas de curto e médio prazos, sem diferença estatisticamente significante nas previsões mais longas.

Apesar de um desinteresse inicial com as conclusões sobre a irrestrita superioridade dos analistas, alguns estudos passaram a reavaliar essa superioridade sob diferentes aspectos e apresentaram evidências questionando-a com base em vantagem de informação e de tempo apresentadas nos estudos de Brown et al. (1982a, 1982b). De acordo com Bradshaw et al. (2012), os estudos que balizaram tal consenso dentro da linha de pesquisa foram realizados com previsões para curto prazo e, portanto, não podem ser extrapolados para a avaliação da superioridade dos analistas em 
previsões de longo prazo. As pesquisas mais recentes indicaram que a superioridade dos analistas não é irrestrita e que a previsão de resultados futuros das empresas por modelos de séries temporais pode ser útil para a tomada de decisão do investidor e para pesquisas em outras áreas do conhecimento (Lorek \& Willinger, 2002; Lacina, Lee \& Xu, 2011; Bradshaw et al., 2012; Grigaliuniene, 2013; Gatsios \& Lima, 2019).

\subsection{Conteúdo informacional das previsões de lucro}

Destacam-se ainda nessa linha recente de pesquisa os estudos sobre o conteúdo informacional das previsões. Cheng, Fan e So (2003), em estudo realizado em Hong Kong, apontaram que as previsões dos analistas e dos modelos de séries temporais apresentam conteúdo informacional para as previsões de resultado das empresas. O estudo foi realizado de 1991 a 1997 e segue a literatura tradicional de superioridade dos analistas, sugerindo maior acurácia dos analistas no curto prazo. Com relação ao conteúdo informacional das previsões dos analistas e dos modelos random walk, Cheng, Fan e So (2003) apontaram que as previsões dos modelos de séries temporais apresentaram maior informação com relação ao lucro atual das empresas nas previsões de longo prazo. Enquanto isso, nas previsões de curto prazo, verificou-se um maior conteúdo informacional nas previsões realizadas pelos analistas.

Lacina, Lee e Xu (2011) compararam a acurácia e o conteúdo informacional de longo prazo dos analistas com os resultados das previsões a partir de modelos random walk com e sem drift. O estudo utilizou dados de 1988 a 2003 com previsões de até cinco anos anteriores e comparou com a mediana das previsões dos analistas. Para a previsão um ano à frente, o trabalho utilizou os dados apresentados pelos analistas de mercado. Os resultados obtidos apontaram que as previsões quatro e cinco anos à frente dos modelos random walk foram superiores às previsões dos analistas. Além disso, o estudo destaca que a superioridade dos analistas declinou para empresas com alta taxa de crescimento dos lucros e com baixa cobertura por parte dos analistas. Ao avaliar o conteúdo informacional das previsões dos analistas e dos modelos random walk, o estudo aponta que as previsões dos modelos de séries temporais apresentaram maior conteúdo informacional com relação ao lucro atual das empresas em previsões de longo prazo. Em contraponto, no curto prazo, verifica-se maior conteúdo informacional nas previsões dos analistas. Vale ressaltar que esses resultados se alinham com os obtidos por Cheng, Fan e So (2003). Alinhado com o que fora destacado por Jegadeesh, Krische e Lee (2004), que os participantes do mercado se interessam em compreender como as atividades dos analistas afetam a eficiência do mercado; e de acordo com os resultados obtidos, o papel dos analistas como intermediários informacionais e como agentes importantes para a eficiência dos mercados foi verificado em previsões de longo prazo.

Diante disso, a literatura aponta que ainda que as previsões dos analistas sejam mais acuradas do que as previsões dos modelos de séries temporais, essas podem conter informações sobre o lucro das empresas, como apresentado por Cheng, Fan e So (2003) e Lacina, Lee e Xu (2011).

A avaliação do conteúdo informacional das previsões dos analistas e dos modelos de séries temporais é proposta nos estudos de Cheng, Fan e So (2003) e Lacina, Lee e Xu (2011). De acordo com esses trabalhos, ainda que os analistas apresentem maior acurácia nas previsões, não se pode subestimar a persistência dos lucros e os modelos random walk, ao utilizar as previsões passadas como base para as previsões futuras, que podem conter informações sobre a memória dos resultados passados nos lucros futuros. Ou seja, mesmo que as previsões dos modelos random walk sejam menos acuradas do que as previsões dos analistas, elas ainda podem ser úteis aos investidores de mercado e aos próprios analistas na avaliação do comportamento futuro dos lucros empresariais. Nesse sentido, a baixa acurácia e o viés de previsão dos analistas no Brasil (LIMA, 2013), o tamanho do mercado acionário brasileiro, menor número de analistas e de empresas listadas, e a alta concentração acionária, podem complementar os resultados já reportados pela literatura prévia - 
especialmente dos estudos realizados em mercados desenvolvidos. Destaca-se também a ausência de pesquisas no contexto brasileiro sobre esse tema. Por fim, baseada na literatura, apresenta-se a hipótese de pesquisa aqui desenvolvida e testada:

$\mathrm{H}_{0}$ : As previsões de lucro dos analistas de mercado apresentam maior conteúdo informacional para a previsão do resultado futuro das empresas brasileiras de capital aberto quando comparadas às previsões dos modelos random walk. Com o intuito de responder o problema de pesquisa e testar a hipótese apresentada, é apresentada a metodologia da pesquisa desenvolvida.

\section{METODOLOGIA}

\subsection{Amostra e Variáveis}

Os dados utilizados neste estudo foram extraídos da plataforma Thomson Reuters ${ }^{\circledR}$, nas bases de dados do I/B/E/S ${ }^{\circledR}$ e Thomson Financial ${ }^{\circledR}$. A amostra completa é composta por 3.751 observações, compreendidas entre 2010 e 2015, e os dados obtidos contemplam as empresas brasileiras de capital aberto. Esse conjunto de observações é composto por previsões de curto e de longo prazos, para um total de 227 empresas. Vale destacar que foram excluídas as observações que não apresentavam dados para as variáveis dependentes e independentes no período analisado, e isso se deve à necessidade de uma base de comparação entre a previsão de lucro realizada pelos analistas e as previsões obtidas pelos modelos random walk. Após a exclusão das observações, a amostra passou a ser composta de 3.479 observações, dividida em 2.014 observações defasadas em um ano, 1.081 com defasagem de dois anos e 384 para três anos. Essas informações podem ser visualizadas na Tabela 1 abaixo.

Tabela 1 - Estatísticas descritivas.

\begin{tabular}{|c|c|c|c|c|c|}
\hline \multicolumn{6}{|c|}{ Amostra Completa } \\
\hline Variável & Observações & Média & D. Padrão & Mín & Máx. \\
\hline ERROANALIST & 3.751 & 0,8413 & 1,1678 & 0,0181 & 4,5125 \\
\hline ERRORW & 3.751 & 0,7591 & 0,9441 & 0,0309 & 3,6496 \\
\hline ERRORWD & 3.751 & 0,8499 & 1,1504 & 0,0303 & 4,6148 \\
\hline \multicolumn{6}{|c|}{ Painel A - defasagem de 1 ano } \\
\hline Variável & Observações & Média & D. Padrão & Mín & Máx. \\
\hline ERROANALIST & 2.015 & 0,7974 & 1,1593 & 0,0181 & 4,5125 \\
\hline ERRORW & 2.015 & 0,7458 & 0,9804 & 0,0309 & 3,6496 \\
\hline ERRORWD & 2.015 & 0,8155 & 1,1666 & 0,0303 & 4,6148 \\
\hline \multicolumn{6}{|c|}{ Painel B - defasagem de 2 anos } \\
\hline Variável & Observações & Média & D. Padrão & Mín & Máx. \\
\hline ERROANALIST & 1.083 & 0,8609 & 1,1550 & 0,0181 & 4,5125 \\
\hline ERRORW & 1.083 & 0,7679 & 0,9208 & 0,0309 & 3,6496 \\
\hline ERRORWD & 1.083 & 0,9019 & 1,1643 & 0,0303 & 4,6148 \\
\hline \multicolumn{6}{|c|}{ Painel C - defasagem de 3 anos } \\
\hline Variável & Observações & Média & D. Padrão & Mín & Máx. \\
\hline ERROANALIST & 653 & 0,9440 & 1,2087 & 0,0181 & 4,5125 \\
\hline ERRORW & 653 & 0,7857 & 0,8658 & 0,0309 & 3,6496 \\
\hline ERRORWD & 653 & 0,8699 & 1,0720 & 0,0303 & 4,6148 \\
\hline
\end{tabular}

Fonte: Elaborado pelos autores.

Para evitar que a presença de outliers interfira na análise dos resultados, foi utilizada a técnica de "winsorização" dos dados, procedimento estatístico que substitui os valores acima ou abaixo de percentis críticos por dados do limite superior ou inferior desses percentis. Essa técnica 
foi aplicada seguindo os métodos descritos nos estudos de Brown et al. (1987), Fried e Givoly (1982) e Lacina, Lee e Xu (2011). Lacina, Lee e Xu (2011) utilizaram na pesquisa duas técnicas para o tratamento dos dados. Além de realizar a "winsorização" dos dados, retiraram os outliers após esse processo. No presente estudo adotou um limite de $5 \%$ para o percentil inferior e de $95 \%$ para o percentil superior. Para a análise da acurácia dos analistas foram utilizados os dados: (i) mediana das projeções realizadas pelos analistas para as empresas brasileiras de capital aberto; e (ii) lucro por ação ajustado das empresas, obtido por meio da base de dados I/B/E/S ${ }^{\circledR}$.

Adicionalmente, para construir as variáveis que medem o erro de previsão dos analistas de mercado e dos modelos random walk para o período de 2010 a 2015, foi necessária a utilização de dados compreendidos entre 2008 e 2015, devido à necessidade de lucros e previsões anteriores para a avaliação da superioridade dos analistas com um, dois e três períodos de defasagem. A Tabela 2 apresenta, de maneira sumarizada, como cada observação de previsão dos analistas foi avaliada. Para a previsão dos modelos random walk apenas foi utilizado o lucro anterior reportado pela empresa para cada defasagem da amostra.

Tabela 2 - Defasagem de previsão dos analistas.

\begin{tabular}{|c|c|c|c|}
\hline Ano & 1 ano - curto prazo & 2 anos - longo prazo & 3 anos - longo prazo \\
\hline 2010 & Março/Jun./Set./Dez. 2010 & Março/Jun./Set./Dez. 2009 & Março/Jun./Set./Dez. 2008 \\
\hline 2011 & Março/Jun./Set./Dez. 2011 & Março/Jun./Set./Dez. 2010 & Março/Jun./Set./Dez. 2009 \\
\hline 2012 & Março/Jun./Set./Dez. 2012 & Março/Jun./Set./Dez. 2011 & Março/Jun./Set./Dez. 2010 \\
\hline 2013 & Março/Jun./Set./Dez. 2013 & Março/Jun./Set./Dez. 2012 & Março/Jun./Set./Dez. 2011 \\
\hline 2014 & Março/Jun./Set./Dez. 2014 & Março/Jun./Set./Dez. 2013 & Março/Jun./Set./Dez. 2012 \\
\hline 2015 & Março/Jun./Set./Dez. 2015 & Março/Jun./Set./Dez. 2014 & Março/Jun./Set./Dez. 2013 \\
\hline
\end{tabular}

Fonte: Elaborado pelos autores.

A variável ERROANALIST foi utilizada para avaliar a acurácia da previsão dos analistas. Sua construção está alinhada com o estudo de Cotter, Tarca e Wee (2012), e encontra-se respaldada na literatura (Lang \& Lundholm, 1996; Hope, 2003; Martinez, 2004; Lacina, Lee \& Xu, 2011; Bradshaw et al., 2012). A variável está descrita na Equação (1):

$$
\text { ERROANALIST }=\left|\frac{F(j, r)-A_{j, r}}{A_{j, r}}\right|
$$

Para as previsões de curto prazo (até um ano de defasagem), a variável ERROANALIST é definida como a diferença absoluta (módulo) entre a mediana da previsão dos analistas $F(j, r)$ e o LPA ajustado anual da empresa j no período da divulgação do resultado $\left(A_{j, r}\right)$, dividida pelo LPA ajustado anual da empresa j no período da divulgação do resultado $\left(A_{j, r}\right)$. O LPA ajustado fora igualmente utilizado por Martinez (2004) e Antônio et al. (2019), e sua utilização é explicada pelo fato de os analistas não incorporarem em suas previsões os itens não recorrentes que afetam o lucro das empresas. A escolha pela mediana em vez da média seguiu como referências estudos anteriores que indicaram que a média sofre maior influência dos dados extremos da amostra (Lang \& Lundholm, 1996; Hope, 2003; Martinez, 2004; Lacina, Lee \& Xu, 2011; Bradshaw et al., 2012).

Para as previsões com mais de um ano de defasagem (classificadas como longo prazo), as estimativas dos analistas são escassas na base de dados brasileira, interferindo na construção da previsão do analista. Diante disso, o presente estudo utilizou a metodologia proposta por Lacina, 
Lee e Xu (2011) e Bradshaw et al. (2012) que utiliza a taxa de crescimento da mediana das estimativas dos analistas de mercado, LTG, disponível na base de dados $\mathrm{I} / \mathrm{B} / \mathrm{E} / \mathrm{S}^{\circledR}$, como apresentado na Equação (2).

A taxa de crescimento é apresentada para cada ano analisado e é acumulada para defasagens maiores. Na previsão com dois anos de defasagem é utilizada a previsão do analista com defasagem de um ano e atualizada pela LTG do próximo período. Para previsões com três anos de defasagem é utilizada a previsão dos analistas com defasagem de um ano e atualizada pela LTG acumulada para o período.

$$
F_{j,(t+\tau)}=F_{j,(t)} \times\left(1+L T G_{(\tau)}\right)
$$

Em que:

$F_{j,(t+\tau)}$ : representa a previsão do analista para a empresa $\mathrm{j}$ da data futura $t+\tau$; dados;

$F_{j,(t)}$ : representa a previsão do analista para a empresa j da data $t$, disponível na base de

LTG: taxa de crescimento do lucro das empresas, mediana do consenso dos analistas;

Dado: $\tau=1$ e 2 .

A variável ERROANALIST foi avaliada com dados referentes aos meses de março, junho, setembro e dezembro anteriores à data da divulgação do resultado fiscal da empresa. Deste modo, foram avaliados quatro diferentes momentos de previsões, contemplando cada período de defasagem. A acurácia dos modelos random walk foi avaliada pela diferença do lucro anterior e o lucro atual divulgado pela empresa (Lacina, Lee \& Xu, 2011; Bradshaw et al. 2012).

Para Bueno (2012) o modelo random walk é definido como um passeio aleatório de tendência estocástica pura, sendo um processo não estacionário. A partir de um modelo random walk entende-se que a melhor estimativa para o resultado futuro é o próprio valor presente. Enquanto isso, de acordo com Brown (1993), essa modelagem apresenta benefícios, pois demanda uma menor quantidade de dados para a realização da estimação. Para pesquisas que não possuem dados disponíveis, é uma alternativa de previsão simples e barata que, dependendo da estrutura de memória da série, pode apresentar resultados relevantes. Essa metodologia é baseada na estrutura de previsão de um modelo random walk, uma vez que, dado o comportamento aleatório do erro, entende-se que a melhor estimativa para o resultado futuro é o próprio valor presente, podendo ser apresentado de acordo com a Equação (3):

$$
Y_{t+1}=Y_{t}+e_{t}
$$

Em que:

$Y_{t+1}:$ representa o valor estimado de determinada série;

$Y_{t}:$ representa o valor atual da série analisada;

$e_{t}:$ representa um erro aleatório.

A escolha dessa metodologia é justificada pelo desenho da pesquisa, que busca comparar as estimativas dos analistas de mercado com modelos de previsão de simples e de fácil utilização pelos agentes (investidores institucionais ou não); e pelo referencial teórico da pesquisa que aponta para o comportamento random walk da série de lucro das empresas, como destacado por Brown (1993). Ainda, essa decisão encontra-se fundamentada nos resultados que indicam que os modelos 
de previsão mais complexos não apresentam necessariamente resultados mais acurados para a previsão de resultado futuro das séries de lucros, como apresentado no estudo de Grigaliuniene (2013). Por fim, essa escolha ainda possibilita a utilização de uma quantidade maior de dados na amostra, uma vez que a previsão necessita apenas da estimativa anterior de lucro da empresa, fato que é importante para a amostra do estudo, dada a quantidade reduzida de dados no caso brasileiro. Ainda com relação ao modelo random walk, pode-se adicionar ao modelo básico uma tendência. Esse ajuste é apresentado na equação (4), como apresentado por Bueno (2012):

$$
Y_{t+1}=Y_{t}+e_{t}(4)
$$

Nesse modelo, $\gamma$ é a constante do modelo. Tal constante é apresentada na literatura como o drift do modelo, denominado, portanto como random walk com drift ou crescimento. Para a linha de pesquisa de previsão de lucros das empresas por meio de modelos random walk, a tendência adicionada ao modelo reflete, de maneira geral, o comportamento da inflação e do crescimento do PIB no período.

O presente trabalho seguiu a metodologia apresentada nos estudos desenvolvidos por Lacina, Lee e Xu (2011) e Bradshaw et al. (2012), ao utilizar varáveis econômicas para a construção do modelo random walk. Nessa construção, o drift do modelo não é uma constante, sendo a previsão multiplicada pela taxa de crescimento em questão. Essa pesquisa utiliza a taxa de crescimento do PIB real brasileiro no período, descontando o IPCA do período, como fator de crescimento para as estimativas. Para a construção da variável de previsão dos modelos random walk com e sem drift, foram construídas as variáveis random walk simples (RWPREV) e random walk com drift (RWDPREV), como apresentadas nas equações (5) e (6). As previsões de tais modelos consideraram apenas os resultados anteriores divulgados pelas empresas.

$$
R W P R E V_{j,(t)}=E P S_{j(t-1)}(5)
$$

Em que:

$E P S_{t-1}$ : representa o lucro por ação da empresa $j$ no período anterior a previsão.

$$
R W D P R E V_{j,(t+\tau)}=E P S_{t-1} \times\left(1+g_{\tau}\right) \quad \in \tau(1,2)
$$

Em que:

$E P S_{t-1}$ : representa o lucro por ação da empresa $j$ no período anterior à previsão;

$g$ : representa a taxa de crescimento real do PIB brasileiro no período (taxa ex-post).

Após a definição do cálculo da previsão RWPREV e RWDPREV com e sem drift, são apresentadas as variáveis ERRORW e ERRORWD, que representam respectivamente o erro de previsão dos modelos random walk com e sem drift - Equações (7) e (8).

$$
E R R O R W_{j, t}=\left|\frac{R W P R E V_{j, t}-A_{j, r}}{A_{j, r}}\right|
$$




$$
E R R O R W D_{j, t}=\left|\frac{R W D P R E V_{j, t}-A_{j, r}}{A_{j, r}}\right|
$$

Em que o erro de previsão é definido pela diferença absoluta entre as estimativas dos modelos random walk - sem drift $\left(R W P R E V_{j, t}\right)$ ou com drift $\left(R W D P R E V_{j, t}\right)$ para a empresa j no período t1 o LPA anual da empresa j no período da divulgação do resultado $\left(A_{j, r}\right)$, dividida pelo LPA anual da empresa j no período da divulgação do resultado $\left(A_{j, r}\right)$. Com o objetivo de comparar as estimativas dos analistas de mercado com as estimativas dos modelos simples de previsão random walk, elas foram calculadas para curto e longo prazo, com defasagens de um até três períodos.

\subsection{Modelo de Conteúdo Informacional}

A avaliação do conteúdo informacional das previsões dos analistas de mercado e dos modelos de séries temporais é proposta nos estudos de Cheng, Fan e So (2003) e Lacina, Lee e Xu (2011). De acordo com esses estudos, ainda que os analistas de mercado apresentem maior acurácia nas previsões, podem subestimar a persistência dos lucros e os modelos random walk, que utilizar a previsão passadas como base para previsões futuras, e podem conter informações sobre a memória dos resultados passados nos lucros futuros.

Ou seja, mesmo que as previsões obtidas por meio dos modelos random walk sejam menos acuradas do que as previsões dos analistas de mercado, as previsões dos modelos de random walk ainda podem ser úteis aos investidores de mercado e aos próprios analistas na avaliação do comportamento futuro dos lucros empresariais. Como apresentado na metodologia, o presente estudo utilizou um modelo de regressão simples com o objetivo de avaliar o conteúdo informacional de cada uma das formas de previsões observadas na pesquisa - previsão dos analistas, previsão dos modelos de random walk simples e previsão do modelo random walk com crescimento. Na equação (9) é reapresentado o modelo utilizado na pesquisa:

$$
\frac{E P S_{t+\pi}}{E P S_{t-1}}-\frac{R W(D) E S P S_{t+\pi}}{E P S_{t-1}}=\alpha+\beta\left(\frac{\operatorname{ANALISESPS}_{t+\pi}}{E P S_{t-1}}-\frac{R W(D) E S P S_{t+\pi}}{E P S_{t-1}}\right)+\varepsilon_{t+\pi}
$$

Onde:

$E P S_{T+\pi}$ : representa o lucro divulgado pela empresa no período $t+\pi$;

$E P S_{t-1}$ : representa o lucro divulgado pela empresa em $t-1$;

$R W E S P S(R W D E S P)_{t+\pi}$ : representa previsão do modelo random walk para o período $t+\pi$;

$\operatorname{ANALISESPS}_{t+\pi}$ : representa previsão dos analistas para o período $t+\pi$;

$\varepsilon_{t+\pi}$ : representa o erro de estimação do modelo.

Caso toda a informação sobre a alteração do lucro realizado sobre o lucro anterior seja explicada pelos analistas de mercado, o coeficiente angular do modelo $\beta$ será igual a 1 . Ao contrário, se a previsão dos analistas não agregar nenhuma informação sobre o lucro futuro, então o $\beta$ será igual a zero.

Caso o $\beta$ esteja entre zero e 1, então ambas as previsões agregam informação sobre o resultado futuro da empresa. Para casos de $\beta$ maior que 1 ou menor que zero, ambas as estimativas possuem informação sobre o lucro futuro, mas os investidores atribuem resultado negativo a uma delas. Ou seja, a magnitude de $\beta$ representa a informação contida nas estimativas dos modelos random walk e nas previsões dos analistas de mercado. 
Como já apresentado, no sentido de facilitar a análise dos resultados, a variável dependente desse modelo é definida como INFDRW e a variável independente como INFIRW. Para o modelo que utiliza as informações dos modelos com crescimento, a variável dependente é tratada por INFDRWD e a variável independente por INFIRWD.

O destaque dessa avaliação é a possibilidade de mesmo que a estimativa dos modelos seja menos acurada, esses podem ser utilizados de maneira complementar na previsão dos resultados futuros das empresas. De maneira sumarizada, a Tabela 3 descreve os alinhamentos metodológicos deste estudo com os adotados como referências metodológicas.

Tabela 3 - Estudos base da metodologia adotada.

\begin{tabular}{|c|c|c|c|c|}
\hline Estudos & Variáveis utilizadas & $\begin{array}{l}\text { Tipo de } \\
\text { análise }\end{array}$ & $\begin{array}{l}\text { Período } \\
\text { de análise }\end{array}$ & Alinhamento metodológico \\
\hline $\begin{array}{l}\text { Bradshaw } \\
\text { et al. } \\
\text { (2012) }\end{array}$ & $\begin{array}{l}\text { Previsões de LPAs e lucros } \\
\text { reportados para 1, } 2 \text { e } 3 \\
\text { anos; } \\
\text { Variáveis das firmas: a) } \\
\text { vendas, b) book-to-market, c) } \\
\text { idade da previsão do LPA } \\
\text { e d) número de analistas. }\end{array}$ & $\begin{array}{l}\text { Quantitativa, } \\
\text { por meio de } \\
\text { regressão } \\
\quad \text { (OLS) }\end{array}$ & $1983-2008$ & $\begin{array}{l}\text { a) Utiliza a taxa de crescimento da } \\
\text { mediana das estimativas dos analis- } \\
\text { tas de mercado, LTG, disponível na } \\
\text { base de dados I/B/E/S } \\
\text { b) A acurácia dos modelos random } \\
\text { walk foi avaliada pela diferença do } \\
\text { lucro anterior e o lucro atual divul- } \\
\text { gado pela empresa }\end{array}$ \\
\hline $\begin{array}{l}\text { Cheng et } \\
\text { al. (2003) }\end{array}$ & $\begin{array}{l}\text { Previsões de LPAs coleta- } \\
\text { dos mensalmente e lucros } \\
\text { reportados anualmente }\end{array}$ & $\begin{array}{l}\text { Quantitativa, } \\
\text { por meio de } \\
\text { regressão } \\
\text { (OLS) }\end{array}$ & $1991-1997$ & $\begin{array}{l}\text { a) Utiliza o modelo de regressão } \\
\text { linear simples. } \\
\text { b) Os LPAs são ajustados aos itens } \\
\text { extraordinários }\end{array}$ \\
\hline $\begin{array}{l}\text { Brown et } \\
\text { al. }(1987 a)\end{array}$ & $\begin{array}{l}\text { Previsões de LPAs coleta- } \\
\text { dos mensalmente e lucros } \\
\text { reportados anualmente }\end{array}$ & $\begin{array}{l}\text { Quantitativa, } \\
\text { por meio de } \\
\text { regressão } \\
\text { (OLS) }\end{array}$ & 1975 - 1979 & $\begin{array}{l}\text { a) Utilização da "winsorização" dos } \\
\text { dados }\end{array}$ \\
\hline
\end{tabular}

Fonte: Elaborado pelos autores.

Após a apresentação da metodologia são apresentados os resultados da pesquisa.

\section{RESULTADOS}

De maneira complementar, estão reportados na Tabela 4 os resultados dos testes de diferença entre médias, considerando as previsões dos analistas e as estimativas dos modelos random walk. Verifica-se que a previsões dos analistas apresentam uma média de erro maior com o aumento da defasagem, partindo de 0,7974 para previsões de um ano, e alcançando 0,944 com uma defasagem de 3 anos. Esses resultados estão alinhados com Gatsios e Lima (2019), e indicam um aumento do erro das previsões dos analistas com o aumento do horizonte temporal. Além disso, para todas as defasagens e anos considerados neste estudo, verificou-se a existência de diferença estatisticamente significante entre a variável ERROANALIST e ERRORW. Ou seja, os resultados reportados sugerem que as previsões obtidas a partir dos modelos random walk são mais precisas do que as previsões realizadas pelos analistas.

Tabela 4 - Teste de diferença de média (erros de estimação).

\begin{tabular}{lcccccccc}
\hline \multicolumn{1}{c}{ Amostra completa } & \multicolumn{2}{c}{ 1 ano } & \multicolumn{2}{c}{ anos } & \multicolumn{2}{c}{ 3 anos } \\
\hline Variável & Média & Teste T & Média & Teste T & Média & Teste T & Média & Teste T \\
\hline ERROANALIST & 0,8413 & & 0,7974 & & 0,8609 & & 0,944 & \\
\hline ERRORW & 0,7591 & $0,0004^{* * *}$ & 0,7458 & $0,0635^{*}$ & 0,7679 & $0,0192^{* *}$ & 0,7857 & $0,0033^{* * *}$ \\
\hline ERRORWD & 0,8499 & 0,6268 & 0,8155 & 0,6893 & 0,9019 & 0,7948 & 0,8699 & 0,1205 \\
\hline
\end{tabular}

Fonte: Elaborado pelos autores. Nível de significância: ${ }^{*} 10 \% ;{ }^{* *} 5 \%$; ${ }^{* *} 1 \%$. 
Para análise dos resultados foi utilizada a metodologia de regressão linear simples (OLS) robusta a heterocedasticidade. Foi utilizada a constante no modelo com o objetivo de adequar o modelo à presença de viés de previsão das estimativas dos analistas de mercado. Essa avaliação é apresentada por Cheng, Fan e So (2003) e Lacina, Lee e Xu (2011).

$\mathrm{Na}$ análise do modelo, Tabela 5, que utiliza a variável INFDRW, os resultados indicam coeficiente positivo e significante a $1 \%$ para a variável INFIRW nas amostras: total, um e dois anos de defasagem. Para a amostra com três anos de defasagem o coeficiente não é significativo. $\mathrm{O}$ coeficiente para a amostra total é de 0,2185 , para a amostra de um ano o resultado é de 0,3346 e para os dados com dois anos de defasagem, 0,0114.

Por esses resultados, nota-se para ambos os períodos de análise que as informações contidas nas previsões dos modelos random walk são relevantes para a previsão de resultado futuro das empresas, uma vez que o coeficiente da variável independente é menor do que 1. 
Tabela 5 - Modelo conteúdo informacional das previsões.

\begin{tabular}{|c|c|c|c|c|c|c|c|c|}
\hline & Total & 1 ano & 2 anos & 3 anos & Total & 1 ano & 2 anos & 3 anos \\
\hline & \multicolumn{4}{|c|}{ INFDRW } & \multicolumn{4}{|c|}{ INFDRW } \\
\hline \multirow{2}{*}{ INFIRW/INFIRWD } & $0,2185^{* * *}$ & $0,3346^{* * *}$ & $0,1140^{* * *}$ & 0,0667 & $0,3441^{* * *}$ & $0,4260^{* * *}$ & $0,2554^{* * *}$ & $0,0795^{* *}$ \\
\hline & $(0,0222)$ & $(0,0361)$ & $(0,0290)$ & $(0,0576)$ & $(0,0342)$ & $(0,0487)$ & $(0,0616)$ & $(2,4700)$ \\
\hline \multirow{2}{*}{ Constante } & $-0,2093^{* * *}$ & $-0,2145^{* * *}$ & $-0,1323^{* * *}$ & $-0,2874^{* * *}$ & $-0,2584^{* * *}$ & $-0,2643^{* * *}$ & $-0,1967^{* * *}$ & $-0,2656^{* * *}$ \\
\hline & $(0,0161)$ & $(0,0219)$ & $(0,0257)$ & $(0,0455)$ & $(0,020)$ & $(0,0266)$ & $(0,0421)$ & $(0,0686)$ \\
\hline Observações & 3.479 & 2.014 & 1.081 & 384 & 3479 & 2014 & 1081 & 384 \\
\hline$R^{2}$ ajustado & 0,0697 & 0,1133 & 0,0346 & 0,0105 & 0,0782 & 0,105 & 0,0436 & 0,0507 \\
\hline Modelo & OLS & OLS & OLS & OLS & OLS & OLS & OLS & OLS \\
\hline
\end{tabular}

Fonte: Elaborado pelos autores. Nível de significância: ${ }^{*} 10 \%$; ${ }^{* *}$ \%; ${ }^{* * *} 1 \%$. Valor entre parênteses: desvio-padrão do coeficiente. 
Evidencia-se que o conteúdo informacional das previsões dos analistas perde intensidade com o aumento da defasagem da previsão - os coeficientes são menores para períodos com defasagem maior e, para o período final com três anos de defasagem, como o coeficiente da variável é não significativo, a interpretação é de que as previsões dos analistas não apresentam conteúdo informacional para previsões com essa defasagem, aumentando assim a relevância das previsões dos modelos random walk.

Ainda pelos resultados apresentados, nota-se maior relevância das previsões dos modelos random walk; mesmo para as estimativas de curto prazo, nota-se um coeficiente mais próximo de 0 do que de 1, pois como discutido na formulação do modelo, valores mais próximos de 1 indicam maior conteúdo informacional por parte dos analistas de mercado, e, consequente, menor conteúdo informacional das previsões dos modelos random walk.

A análise do modelo que utiliza a variável INFDRWD apresenta resultados que indicam coeficiente positivo e significante a $5 \%$ para a variável INFIRWD nas amostras: total, um, dois e três anos de defasagem. O coeficiente para a amostra total é de 0,3441 , para a amostra de um ano o resultado é de 0,4260, e para os dados com dois e três anos de defasagem 0,2554 e 0,0795, respectivamente.

A interpretação é semelhante ao observado com relação ao modelo que utiliza informações da metodologia random walk simples para a previsão de lucro futuro das empresas. Os coeficientes e a significância dos modelos reforçam a maior acurácia dos modelos random walk simples sobre os modelos de random walk com crescimento, uma vez que para a variável SPRWD o conteúdo informacional dos analistas é maior. Deste modo, a relevância da previsão dos analistas é maior nesta comparação.

Ainda assim, são apresentados na Tabela 4 os resultados dos modelos que avaliam o conteúdo informacional das previsões nos meses de dezembro, setembro, junho e março, para os três períodos de defasagem. Os resultados complementam a análise dos dados anuais e reforçam que para períodos de curto prazo. Verifica-se maior conteúdo informacional na previsão dos analistas de mercado; sendo que com o aumento da defasagem a previsão dos analistas perde conteúdo informacional com relação ao lucro futuro.

Os resultados confirmam ainda a significância do conteúdo informacional dos modelos random walk, com maior magnitude do modelo simples. Por fim, confirma-se a avaliação sobre a existência de viés de previsão das estimativas, dada a significância da constante nos modelos.

Os resultados das constantes dos modelos são significantes, indicando a presença de viés de previsão nas estimativas para o caso brasileiro no período analisado. Fato destacado nas pesquisas brasileiras sobre a qualidade da previsão das estimativas dos analistas de mercado no Brasil (Martinez, 2007).

Esses resultados rejeitam a hipótese proposta nessa pesquisa e aumentam o indicativo de que a superioridade dos analistas não é irrestrita para o caso brasileiro, reforçando a avaliação de que as previsões dos modelos random walk são fundamentais na decisão para os usuários dessa informação no Brasil. Nota-se que o conteúdo informacional das previsões dos analistas vai perdendo intensidade conforme o aumento da defasagem da previsão, não sendo significante para o período final - três anos de defasagem. Além disso, para os períodos avaliados verifica-se maior conteúdo informacional nas previsões dos modelos random walk para curto e longo prazo.

Esse resultado se alinha aos achados de Lacina, Lee e Xu (2011) e de Cheng, Fan e So (2003), que indicam conteúdo informacional para ambas as formas de previsão. Contudo, o resultado se afasta dos achados internacionais e se alinha com as evidências encontradas nas avaliações das hipóteses anteriores, ao indicar maior conteúdo informacional nas previsões dos modelos random walk para curto e longo prazo; apresentando evidências de que para períodos com defasagem 
de três anos não se verifica significância para o conteúdo informacional da previsão dos analistas de mercado.

Esse resultado sugere uma ineficiência dos analistas de mercado na transformação das vantagens de tempo e informação para uma maior acurácia de previsão com relação aos modelos de séries temporais. Em função dos resultados obtidos, no ambiente brasileiro o papel dos analistas de mercado como agentes de redução da assimetria informacional e consequente aumento da eficiência dos mercados é limitado. Essa ineficiência pode ser explicada pelas características do mercado de capitais brasileiro. Os achados da pesquisa confirmam as evidências apresentadas por Lima (2015) - menor acurácia dos analistas brasileiros comparados aos dados apresentados nos estudos que avaliam mercados internacionais, pois para o caso brasileiro a superioridade dos analistas não é irrestrita, mesmo para curto prazo.

De posse desses resultados fica evidente a necessidade de pesquisas futuras na área sobre a previsão de resultados das empresas brasileiras de capital aberto, pois como destacado ao longo do trabalho, essas informações são utilizadas por investidores na decisão de alocação de portfólio, e segundo os resultados encontrados, é indicada para esses usuários a utilização de estimativas alternativas de previsão aos analistas de mercado para previsões com defasagem maior de tempo e para alguns grupos específicos da amostra. Ainda, destaca-se que essas estimativas são mais baratas e fáceis de utilizar, estando disponíveis para todas as empresas e não apenas para empresas cobertas pelos analistas de mercado, possibilitando assim uma redução do risco de investimento, e consequentemente afetando a eficiência do mercado de capitais brasileiro. Por fim, é necessário expandir a avaliação sobre essas duas formas de previsão de resultados futuros, previsão de analistas e previsão de modelos de séries temporais.

\section{CONCLUSÃO}

A pesquisa apresenta uma avaliação sobre o conteúdo informacional das previsões dos analistas em comparação às previsões obtidas por meio dos modelos random walk. O estudo parte dos trabalhos que apontaram superioridade dos analistas na previsão dos lucros futuros das empresas, indicando que eles possuem vantagem informacional e de tempo em relação às previsões obtidas por meio dos modelos de séries temporais, como apontado por Brown et al. (1982a), Brown et al.(1982b), Fried e Givoly (1982), Hopwood e Mckeown (1982) e O’brien (1988).

Embora haja essa concepção inicial, novos estudos reavaliaram a superioridade dos analistas com relação aos modelos de séries temporais. Essas pesquisas destacaram que a superioridade dos analistas não é irrestrita e depende de fatores associados a: defasagem da previsão, quantidade de analistas que realizam a cobertura das empresas, dispersão das estimativas dos analistas, variabilidade dos resultados, resultados positivos ou negativos, tamanho da empresa e idade no mercado de capitais (Lacina, Lee \& Xu, 2011; Bradshaw et al., 2012; Lorek \& Pagach, 2014; Ball \& Ghysels, 2017). Adicionalmente, Cheng, Fan e So (2003) e Lacina, Lee e Xu (2011) apontaram que o conteúdo informacional das previsões dos modelos de séries temporais foi maior, associando o resultado à persistência dos lucros.

Diante disso, este trabalho buscou ampliar a literatura sobre a superioridade das previsões dos analistas, buscando responder ao seguinte problema de pesquisa: qual o conteúdo informacional das previsões dos analistas de mercado e dos modelos random walk na previsão de lucro das empresas brasileiras não financeiras de capital aberto?

$\mathrm{Na}$ consecução da pesquisa foram avaliadas as empresas brasileiras de capital aberto com dados disponíveis entre 2010 e 2015. Para tanto, foram utilizadas informações obtidas por meio da plataforma Thomson Reuters ${ }^{\circledR}$, mais especificamente nas bases de dados do I/B/E/S ${ }^{\circledR}$ e Thomson Financial ${ }^{\circledR}$. Os resultados apontaram maior relevância das previsões randon walk quando comparadas com as previsões dos analistas, esse resultado também ocorreu nas previsões de curto prazo. 
Adicionalmente, verificou-se que o conteúdo informacional dos analistas perde a intensidade com o aumento da defasagem.

Esses resultados estão alinhados com Cheng, Fan e So (2003) e Lacina, Lee e Xu (2011), que indicaram conteúdo informacional para ambas as formas de previsão. Contudo, o resultado se afasta dos achados internacionais, ao indicar maior conteúdo informacional nas previsões dos modelos random walk para curto e longo prazos. As evidências apresentadas indicaram que em períodos com maior defasagem não se verificou significância para o conteúdo informacional das previsões dos analistas. Esse resultado sugere uma ineficiência dos analistas na transformação das vantagens de tempo e informação para uma maior acurácia de previsão com relação aos modelos de séries temporais. Nesse sentido, os analistas não contribuem para a redução da assimetria informacional do mercado de capitais brasileiro.

Essa ineficiência pode ser explicada pela baixa acurácia da previsão dos analistas para o mercado de capitais brasileiro ( Lima 2013) e pelas características do mercado de capitais brasileiro, como o tamanho do mercado acionário e o número reduzido de analistas e de empresas listadas em bolsa; ou seja, o atual estágio de desenvolvimento do mercado acionário brasileiro pode ter um papel fundamental nos resultados, de acordo com as características apontadas por Lacina, Lee \& Xu (2011), Bradshaw et al. (2012), Lorek, e Pagach (2014) e Ball e Ghysels (2017).

A partir dos resultados obtidos fica evidente a necessidade de pesquisas futuras na área sobre a previsão de resultados das empresas brasileiras de capital aberto. Destaca-se que as estimativas random walk são mais baratas e fáceis de utilizar, estando disponíveis para todas as empresas e não apenas para empresas cobertas pelos analistas de mercado, possibilitando assim uma redução do risco de investimento. Por fim, é necessário expandir a avaliação sobre essas duas formas de previsão de resultados futuros, previsão de analistas e previsão de modelos de séries temporais. Os resultados deste estudo estão limitados às características institucionais do mercado acionário brasileiro entre o período considerado, que está compreendido entre 2010 e 2015. Adicionalmente, as pesquisas futuras podem explorar em que condições e situações as previsões dos analistas são superiores (ou inferiores) àquelas obtidas pelos modelos de séries temporais.

\section{REFERÊNCIAS}

Antônio, R. M., Lima, F. G., \& Pimenta Júnior, T. (2015). Stock recommendations and investment portfolio formation: a study in the Brazilian market. Contaduría y Administración, 60, 874892. doi: https://doi.org/10.1016/j.cya.2015.07.010.

Antônio, R., Rathke, A., Moraes, M., \& Ambrozini, M. (2018). The Effect of Trade Volume on Analysts' Shares Recommendation Choices. Brazilian Review Of Finance, 15(2), 287-311. doi: http://dx.doi.org/10.12660/rbfin.v15n2.2017.67390.

Antônio, R. M., Lima, F. G., dos Santos, R. B., \& Rathke, A. A. T. (2019). Use of Derivatives and Analysts' Forecasts: New Evidence from Non-financial Brazilian Companies. Australian Accounting Review, 29(1), 220-234. doi: https://doi.org/10.1111/auar.12268.

Ball, R. T., \& Ghysels, E. (2017). Automated Earnings Forecasts: Beat Analysts or Combine and Conquer?. Management Science, 64(10), 4936-4952. doi: https://doi.org/10.1287/mnsc.2017.2864.

Beiruth, A. X. (2012). Avaliação da utilização e precificação de modelos contábeis e de analistas no mercado brasileiro. Master's Dissertation, Faculdade de Economia, Administração e Contabilidade, University of São Paulo, São Paulo. doi: 10.11606/D.12.2012.tde-05112012-174920. 
Boff, L. H., Procianoy, J. L., \& Hoppen, N. (2006). O uso de informações por analistas de investimento na avaliação de empresas: à procura de padrões. Revista de Administração Contemporânea, 10(4), 169-192. doi: http://dx.doi.org/10.1590/S1415-65552006000400009.

Bradshaw, M. T., Drake, M. S., Myers, J. N., \& Myers, L. A. (2012). A re-examination of analysts' superiority over time-series forecasts of annual earnings. Review of Accounting Studies, 17(4), 944-968. doi: https://doi.org/10.1007/s11142-012-9185-8.

Branson, B. C., Lorek, K. S., \& Pagach, D. P. (1995). Evidence on the Superiority of Analysts Quarterly Earnings Forecasts for Small Capitalization Firms. Decision Sciences, 26(2), 243-263. doi: https://doi.org/10.1111/j.1540-5915.1995.tb01428.x.

Brown, L. D. (1993). Earnings forecasting research: its implications for capital markets research. International journal of forecasting, 9(3), 295-320. doi: https://doi.org/10.1016/01692070(93)90023-G.

Brown, L. D., Hagerman, R. L., Griffin, P. A., \& Zmijewski, M. E. (1987a). An evaluation of alternative proxies for the market's assessment of unexpected earnings. Journal of Accounting and Economics, 9(2), 159-193. doi: https://doi.org/10.1016/0165-4101(87)90004-8.

Brown, L. D., Richardson, G. D., \& Schwager, S. J. (1987b). An information interpretation of financial analyst superiority in forecasting earnings. Journal of Accounting Research, 49-67. doi: https://doi.org/10.2307/2491258.

Bueno, R. D. L. da S. (2012). Econometria de séries temporais. São Paulo: Cengage Learning.

Cheng, J. W., Fan, D. K., \& So, R. W. (2003). On the performance of naïve, analyst and composite earnings forecasts: Evidence from Hong Kong. Journal of International Financial Management \& Accounting, 14(2), 146-165. doi: https://doi.org/10.1111/1467-646X.00093.

Cotter, J., Tarca, A., \& Wee, M. (2012). IFRS adoption and analysts' earnings forecasts: Australian evidence. Accounting \& Finance, 52(2), 395-419. doi: https://doi.org/10.1111/j.1467629X.2010.00392.x.

Dalmácio, F. Z., Lopes, A. B., Rezende, A. J., \& Sarlo Neto, A. (2013). Uma análise da relação entre governança corporativa e acurácia das previsões dos analistas do mercado brasileiro. RAM. Revista de Administração Mackenzie, 14(5), 104-139. doi: https://dx.doi.org/10.1590/S167869712013000500005 .

Eid Jr., W.; Rochman, R. R. (2006). Analysts' recommendations in Brazil: Do they add value? Balas: Peru.

Esteter, B., Pedreira, E. B., \& Barros, L. A. B. D. C. (2011). Análise dos Erros de Previsão em Empresas de Capital Aberto para os Mercados de Argentina, Brasil, Chile e México entre os Anos de 2003 a 2009. In II Congresso Nacional de Administração e Ciências ContábeisAdCont 2011. 
Fama, E. F. (1970) Efficient capital markets: A review of theory and empirical work, Journal of Finance, 25, 383-417.

Fried, D., \& Givoly, D. (1982). Financial analysts' forecasts of earnings: A better surrogate for market expectations. Journal of Accounting and Economics, 4(2), 85-107. doi: https://doi.org/10.1016/0165-4101(82)90015-5.

Gatsios, R. C., \& Lima, F. G. (2014). Acurácia dos analistas: Impacto da adoção do padrão IFRS sobre a qualidade preditiva da informação contábil no Brasil. XIV Encontro Brasileiro de Finanças, Recife. Anais. Recife.

Gatsios, R. C.; Lima, F. G. (2019). Reavaliação da superioridade dos analistas na previsão de resultado futuro das empresas brasileiras de capital aberto. XIX ENCONTRO BRASILEIRO DE FINANÇAS, Rio de Janeiro. Anais. Rio de Janeiro.

Griffin, P. A. (1977). The time-series behavior of quarterly earnings: preliminary evidence. Journal of Accounting Research, 1, 71-83. doi: https://doi.org/10.2307/2490556.

Grigaliūnienè, Ž. (2013). Time-series models forecasting performance in the Baltic stock market. Organizations and Markets in Emerging Economies, 4(07), 104-120.

Hall, J. L., \& Tacon, P. B. (2010). Forecast accuracy and stock recommendations. Journal of Contemporary Accounting \& Economics, 6(1), 18-33. doi: https://doi.org/10.1016/j.jcae.2010.04.003.

Hope, O. K. (2003). Disclosure practices, enforcement of accounting standards, and analysts' forecast accuracy: An international study. Journal of accounting research, 41(2), 235-272. doi: https://doi.org/10.1111/1475-679X.00102.

Hopwood, W. S., \& McKeown, J. C. (1990). Evidence on Surrogates for Earnings Expectations Within a Capital Market Context. Journal of Accounting, Auditing \& Finance, 5(3), 339-363.

Jegadeesh, N., Kim, J., Krische, S. D., \& Lee, C. M. (2004). Analyzing the analysts: When do recommendations add value?. The journal of finance, 59(3), 1083-1124. doi: https://doi.org/10.1111/j.1540-6261.2004.00657.x.

Kothari, S. P. (2001). Capital markets research in accounting. Journal of accounting and economics, 31(1-3), 105-231. doi: https://doi.org/10.1016/S0165-4101(01)00030-1.

Kross, W., Ro, B., \& Schroeder, D. (1990). Earnings expectations: The analysts' information advantage. Accounting Review, 461-476.

Lacina, M., Brian Lee, B., \& Zhaohui Xu, R. (2011). An evaluation of financial analysts and naïve methods in forecasting long-term earnings. In Advances in business and management forecasting (pp. 77-101). Emerald Group Publishing Limited.

Lang, M. H., \& Lundholm, R. J. (1996). Corporate disclosure policy and analyst behavior. Accounting review, 467-492. 
Lee, C. M. C. Earnings news and small traders: An intraday analysis. Journal of Accounting and Economics, v. 15, n. 2-3, p. 265-302, 1992. doi: https://doi.org/10.1016/0165-4101(92)90021-S.

Lima, F. G. (2012). Modelos de previsão de séries temporais financeiras com combinação de filtros de Kalman e Wavelets. Tese de Livre Docência, Faculdade de Economia, Administração e Contabilidade de Ribeirão Preto, Universidade de São Paulo, Ribeirão Preto. doi: 10.11606/T.96.2012.tde-27022012-223030.

Lima, G. A. S. F. (2013). A relação do acompanhamento dos analistas com características de valuation das empresas brasileiras. Tese de Livre Docência, Faculdade de Economia, Administração e Contabilidade, Universidade de São Paulo, São Paulo. doi:10.11606/T.12.2014.tde14112014-151023.

Lima, M. P., \& de Souza Almeida, V. (2015). Os analistas sell-side fazem boas previsões de preçosalvo no Brasil? Revista Brasileira de Finanças, 13(3), 365-393. doi: http://dx.doi.org/10.12660/rbfin.v13n3.2015.35208.

Lorek, K. S., \& Willinger, G. L. (2002). An analysis of the accuracy of long-term earnings predictions. Advances in Accounting, 19, 161-175. doi: https://doi.org/10.1016/S08826110(02)19009-2.

Lorek, K. S., \& Pagach, D. P. (2014). Analysts versus Time-Series Forecasts of Quarterly Earnings: A Maintained Hypothesis Revisited. Available at SSRN 2406013.

Martinez, A. (2004). Analisando os analistas: estudo empírico das projeções de lucros e das recomendações dos analistas do mercado de capitais para as empresas brasileiras de capital aberto. Doctoral dissertation. Fundação Getúlio Vargas, São Paulo, SP, Brazil.

Martinez, A. L. (2007). Otimismo e viés de seleção dos analistas. BBR-Brazilian Business Review, 4(2), 104-118. doi: http://dx.doi.org/10.15728/bbr.2007.4.2.2.

Martinez, A. L., \& Dumer, M. (2014). Adoption of IFRS and the Properties of Analysts' Forecasts: The Brazilian Case. Revista de Contabilidade e Organizações, 8(20), 3-16. doi: https://doi.org/10.11606/rco.v8i20.55459.

O'brien, P. C. (1988). Analysts' forecasts as earnings expectations. Journal of Accounting and Economics, 10(1), 53-83. doi: https://doi.org/10.1016/0165-4101(88)90023-7.

Paulo, E., Lima, G. A. S. F. D., \& Lima, I. S. (2006). A influência da cobertura dos analistas financeiros sobre o gerenciamento de resultados das companhias abertas brasileiras. Anais.

Ramnath, S., Rock, S., \& Shane, P. (2008). The financial analyst forecasting literature: A taxonomy with suggestions for further research. International Journal of Forecasting, 24(1), 34-75. doi: https://doi.org/10.1016/j.ijforecast.2007.12.006.

Schipper, K. (1991). Analysts' forecasts. Accounting horizons, 5(4), 105-121. 
Da Silva, H. R. (1998). A capacidade previsionária no mercado acionário brasileiro-Um estudo focado nas previsões dos analistas de investimentos. Encontro da ANPAD, 22. 